\title{
The Shiraz Adult Respiratory Disease Study, a Population-Based Survey: Rationale, Design and Methods
}

\author{
Seyed Masoom Masoompour, ${ }^{1}$ Hamideh Mahdaviazad,, ${ }^{2,}$ Seiyed Mohammad Ali Ghayumi, ${ }^{3}$ and
}

\author{
Mehrab Sayadi ${ }^{4}$ \\ ${ }^{1}$ MD Associate Professor of Internal Medicine, Pulmonologist,Non Communicable Diseases Research Center, Shiraz University of Medical Sciences, Shiraz, IR Iran \\ ${ }^{2} \mathrm{MD}$, Assistant Professor of Community Medicine, Poostchi Ophtalmology research center, Shiraz University of Medical Sciences, Shiraz, IR Iran \\ ${ }^{3} \mathrm{MD}$, Professor of Internal Medicine, Pulmonologist, Department of Internal Medicine, Shiraz University of Medical Sciences, Shiraz, IR Iran \\ ${ }^{4} \mathrm{PhD}$ Student, Student Research Committee, Medicine School, Department of Biostatistics, Shiraz University of Medical Sciences, Shiraz, IR Iran
}

Corresponding author: Hamideh Mahdaviazad, Assistant professor of Community and Preventive Medicine, Shiraz University of Medical Sciences, P.O. Box: 7193634154, Shiraz, IR Iran. Tel: +98-7136234504, Fax: +98-7136234504, E-mail: mahdavih@sums.ac.ir

Received 2017 February 11; Accepted 2017 February 27.

\begin{abstract}
Background: Despite preventable and curable nature of chronic obstructive respiratory diseases (CORDs), these are still remains as a growing problem, both in developed and developing countries. One of the major reasons for this ignorance is lacking of precise information on the prevalence of CORDs and their major determinants.

Objectives: This protocol study aimed at providing a population-based data for the prevalence of CORDs and exploring its socioeconomic determinants in adult population of Shiraz, Iran.

Methods: This adult respiratory disease study was designed as a cross sectional survey among urban and suburban population of Shiraz, Iran, during August to December 2015. The local ethics committee of Shiraz University of Medical Sciences authorized the protocol, which adhered to the tenets of the declaration of Helsinki (1975). The study included more than 6,000 noninstitutionalized inhabitants older than 20 years, from the 9 municipal districts of Shiraz city. The sample consisted of $0.5 \%$ of the 1,219,237 total inhabitants in the survey area. The sample selection was based on the stratified random sampling method proportionate to the number of municipal districts and strata. After obtaining official permissions, trained interviewers visited the selected homes and completed the data collection form and main questionnaire. Following the completion of recruitment and an initial analysis process, we called and invited the participants with the COPD diagnosis score more than 19.5 to pass the spirometry test in the clinic. The main questionnaire that covered respiratory symptoms was developed from prior validated questionnaires that had been used in several studies. Data were analyzed using the SPSS software version 15.0 (SPSS Inc, Chicago, Illinois, USA). A P-value of less than 0.05 was considered as statistically significant.

Results: In this protocol study, we shared our standard protocol considering perfect selection of the study samples and precise examination procedure with effective quality assurance strategies.

Conclusions: We anticipate that future reports from the Shiraz adult respiratory disease study would estimate the prevalence of CORDs and their related determinants in the community. Moreover, it will identify patients who should be targeted by respiratory disease prevention programs.
\end{abstract}

Keywords: Asthma, Adult, Chronic Obstructive Pulmonary Disease, Design, Prevalence, Respiratory Disease

\section{Background}

Chronic obstructive respiratory diseases (CORDs) are a substantial public health problem (1). Despite their preventable and curable nature, CORDs including asthma and the chronic obstructive pulmonary disease (COPD) are still a growing problem, both in developed and developing countries (2).

As the fifth major cause of death, the prevalence of COPD was estimated to be $5.2 \%$ based on the physician diagnosis and $15.1 \%$ based on lung function testing among US adults $(3,4)$. Also, there are 300 million asthmatic patients worldwide who are responsible for $1 \%$ of the total burden of diseases and 250,000 deaths per year (4-6).

In Iran, according to the latest reports, the prevalence rates of asthma and COPD were estimated to be $10.80 \%$ and $9.2 \%$, respectively $(6,7)$. Due to the aging population, social industrialization and changes in the pattern of tobacco consumption, the prevalence of CORDs has been increased in developing countries like Iran $(3,4,6)$. Despite increase in the prevalence of this preventable disease, health policy makers and governments have not paid enough attention to these issues. One of the major reasons for this ignorance is lacking the precise information on the CORD prevalence and its major determinants $(8,9)$. There are scanty population-based surveys on the prevalence of 
CORDs in adult population of Shiraz, and there is no study on the prevalence of CORDs in adult population and its relation with socioeconomic determinants.

This study was conducted to provide a populationbased data for the prevalence of CORDs and explore its association with socioeconomic determinants in adult population of Shiraz, Iran.

\section{Objectives}

The present study was conducted to measure the prevalence rates of COPD and asthma and their socioeconomic determinants, and also to assess the sensitivity and specificity of the COPD diagnosis questionnaire compared with the lung function testing as the gold standard.

\section{Methods}

\subsection{Overview}

This cross sectional survey was conducted on urban and suburban population of Shiraz city, the capital of Fars Province, Iran, from August to December, 2015.

\subsection{Ethical Consideration}

The local ethics committee of Shiraz University of Medical Sciences approved the protocol, which adhered to the tenets of the declaration of Helsinki (1975). After individuals were notified of the study objectives, voluntary participation and right to withdraw, oral informed consent was obtained from them. The confidentiality of all personal data was preserved.

\subsection{Study Population and Sample Size Estimation \\ 3.3.1. Shiraz Population}

Shiraz city is a capital of Fars province, Iran. The 2011 census recorded Fars region's population as 4.59 million that 1.7 million of them are living in the Shiraz city and marginal zones (10). The sampling frame was individuals of the urban and suburban zones of the Shiraz. Table 1 demonstrates the Shiraz population distention in the 9 municipal districts based on the 2011 census data. Accordingly, the study included more than 6000 noninstitutionalized inhabitants 20 and older than 20 years from the 9 municipal districts of Shiraz, Iran. The sample consisted of $0.5 \%$ of the $1,219,237$ total inhabitants aged $\geq 20$ years in the survey area.

\subsection{Inclusion Criteria}

All noninstitutionalized adults $\geq 20$ years in Shiraz, who had sufficient ability to cooperate well during the interview and further examinations were included in the study.

\subsection{Exclusion Criteria}

Inhabitants did not consent to participate were excluded from the study.

\subsection{Sample Size}

For sample size calculation, a complete literature search was performed. There was no population-based study about the prevalence of CORDs in Shiraz, but the prevalence rates of asthma and COPD were reported 10.8 and 9.2, respectively, in recent studies in Tehran $(6,7)$. Considering these reports the prevalence of CORDs was estimated to be approximately $9 \%$ in our study population.

Using the formula;

$\mathrm{N}=\left(\mathrm{z}_{1-\alpha / 2}\right)^{*} \mathrm{p}(1-\mathrm{p}) / \mathrm{d}^{2}$

A minimum sample of 5593 subjects were required based on the $Z=1.96$ (the desired level of confidence $95 \%$ ), margin of error $=0.75 \%$ and $\mathrm{P}=9 \%$. Considering a participating rate of $90 \%$, the final sample size was calculated to be 66152 subjects.

\subsection{Sampling Plan}

The target population was defined as all urban and suburban citizens, aged $\geq 20$ years, who reside in Shiraz city in the year of study. The sample was selected based on the stratified random sampling method proportionate to the number of municipal districts and strata.

The sampling was performed in two stages:

- First stage: stratification

Considering the 9 municipal districts of Shiraz city and stratification of the sample, we selected the participants. Proportional to the total number of the sample size, household size, and logistical facilities, the appropriate number of blocks in each municipal district was calculated using the Microsoft Excel 2007 software (Table 1).

- Second stage: random sampling

After identifying the index household in each block, the interviewer enumerates 10 neighbor households systemically by the clock-wise rotation. If there is more than one eligible person in selected households, the Kish selection method was used to choose the right participant.

\subsection{Recruitment Process and Questionnaires}

The recruitment process was started with necessary coordination to obtain official permission to visit homes. By presenting their identification cards, interviewers introduced themselves and explained the project to the main member of the households; eligible members were invited to complete the questionnaires. At the end of the interview, we asked about the participants' cooperation to perform spirometry if necessary for proving the respiratory disease. Following the completion of recruitment and an 
Table 1. Distribution of the Studied Population and Participation Classified by the Nine Municipal Districts of Shiraz City (2011 Census)

\begin{tabular}{|c|c|c|c|c|}
\hline Districts & Population $^{\mathrm{a}}$ & Households & Sample & Participation \\
\hline 1 & $235,262(13.8)$ & 63,584 & 860 & 858 \\
\hline 2 & $243,083(14.3)$ & 65,698 & 888 & 877 \\
\hline 3 & $239,030(14.1)$ & 64,603 & 873 & 866 \\
\hline 4 & $246,160(14.5)$ & 66,530 & 899 & 855 \\
\hline 5 & $175,847(10.3)$ & 47,526 & 638 & 633 \\
\hline 6 & $180,669(10.6)$ & 48,829 & 658 & 652 \\
\hline 7 & $195,434(11.5)$ & 52,820 & 715 & 705 \\
\hline 8 & $69,206(4.1)$ & 18,704 & 260 & 247 \\
\hline 9 & $115,996(6.8)$ & 31,350 & 423 & 416 \\
\hline Total & $1,700,687(100)$ & 459,645 & 6214 & 6109 \\
\hline
\end{tabular}

${ }^{\mathrm{a}}$ Values are expressed as No. (\%).

initial analysis process, we called and invited the participants with the COPD diagnosis score more than 19.5 (11) to pass the spirometry test in the clinic (Figure 1).

\subsection{Nonresponse}

Following the initial invitation, we tried two other episodes of recruitment, subjects who did not attend the interview process or spirometry test were considered as nonrespondents in this study.

\subsection{Questionnaire}

The main questionnaire that covered respiratory symptoms was developed from prior validated questionnaires that had been used in several studies $(11,12)$. Demographic factors including age, gender, self-reported weight and height and marital status were recorded. The educational level, occupation, residency location and income were being considered as a proxy of the socioeconomic status of each household. Residential areas were classified as urban and suburban districts. Self-reported education was measured by asking each subject about his/her educational level. Finally, responses were combined into three categories including basic, secondary, and higher educational levels. Household income was defined as a sum of all family member income, which received during one year. The classification was based on the median income of all households in Iran.

\subsection{Definitions}

\subsubsection{Asthma}

We considered current asthma if at least one of the following factors presents in the last year: 1) awakening by an attack of shortness of breath, cough, or chest tightness without any identifiable cause, 2) having an asthma attack, 3) currently using medication for asthma and 4) having wheezing or whistling in the chest not associated with a cold or the flu (12). Ever diagnosed asthma was defined as responding "yes" to the question "Has a doctor ever said that you had asthma?"

\subsection{Chronic Obstructive Pulmonary Disease}

A diagnosis of COPD should be considered in each subject with symptoms of cough, productive sputum, dyspnea and history of exposure to risk factors. According to the COPD diagnosis questionnaire, participants who have the COPD diagnosis score more than 19.5 will be candidates to performing spirometry (11). Airway obstruction and its severity are defined as the forced expiratory volume 1(FEV1)/ forced vital capacity (FVC) of lower than 70\% based on the revised GOLD criteria (13).

\subsection{Cigarette Smoking}

Cigarette smoking was defined as active smokers who smoke at the present time and past smokers those who had previously quit smoking. In all participants, duration and number of per day cigarette smoking was recorded.

\subsection{Waterpipe Tobacco Smoking}

Waterpipe smoking was defined as active water-pipe smokers who smoke daily or more frequent at the present time and past smokers those who had previously quit smoking (abstinence from smoking). In all participants, the amount of usage per head of tobacco and duration of smoking in years were assessed. 
Epidemiologic field work

6,214 eligible inhabitants of age $\geq 20$ years selected from 9 municipal districts in urban and suburban areas of the ... city

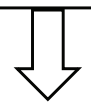

Door- to door visits

Informed consents, demographic data, socio- economic status, asthma, allergic rhinitis and COPD diagnosis questionnaire

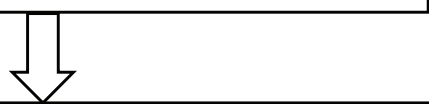

6,109 inhabitants of aged more than 20 years

Completed the questionnaires

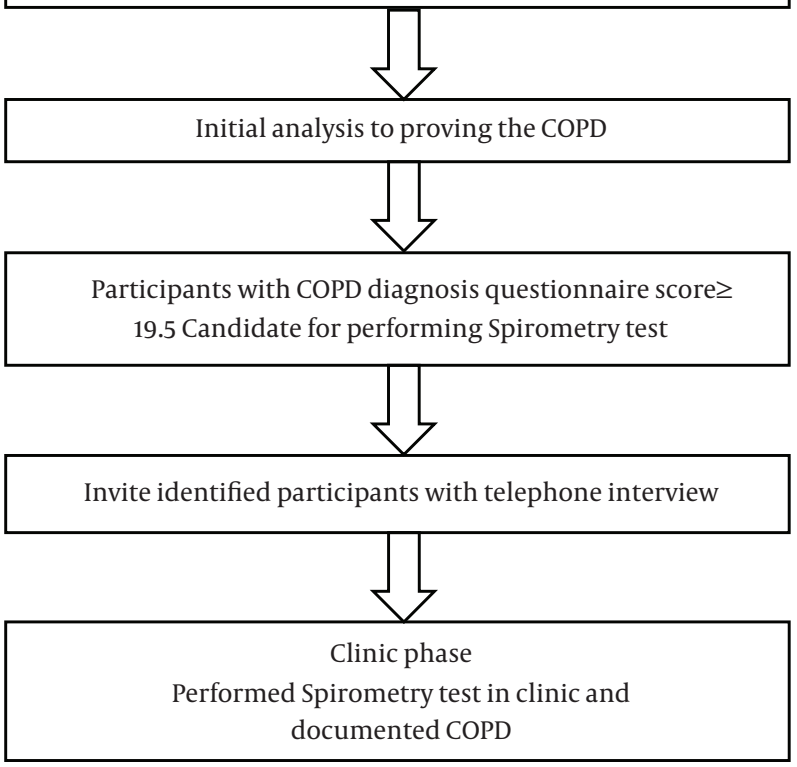

Figure 1. The Process of the Shiraz Adult Respiratory Disease Study

\subsection{Second-Hand Tobacco Smoke}

Second-hand smoke was assessed by whether or not they frequently inhaled waterpipe or cigarette smoke at or outside the home.

\subsection{Spirometry}

Spirometry was done by a specially trained technician in the clinic according to the American thoracic society (ATS) recommendations (14). Spirometry was performed before and after the administration of $200 \mathrm{mg}$ ( 2 puffs) of salbutamol using the spirometer (ZAN 100; Oberthulba, Germany) for any subjects suspicious to have obstructive lung diseases. The primary spirometry measurements in- cluding the FEV1 and the FVC, and FEV1/FVC were measured and analyzed.

\subsection{Quality Assurance}

Before starting the project, several training courses were carried out for the study staff about the study purposes, sample selection method, interview, questionnaires and technical skills needed to conduct the protocol. During the study period (approximately 6 months) the accuracy of interviews were controlled and verified by project observers every week. In addition, the validity of the data was reviewed periodically; the director verified $10 \%-15 \%$ of the questionnaires to monitor the data precision in each cluster and feedback was given to the observers monthly. The spirometry instrument was calibrated daily by trained operators using the manufacturer's guidelines.

\subsection{Data Handling and Statistical Analysis}

\subsubsection{Data Entry and Editing}

On completion of interviews on each block, data were entered and checked. At the clinic, relevancy of the spirometry data has been evaluated by the supervisor. Missing or wrong data were obtained after evaluating the process and consulting with related interviewer. During data entry, the completeness and consistency of the questionnaires were checked by the data entry software.

\subsubsection{Statistical Analysis}

We used the SPSS software version 15.0 (SPSS Inc, Chicago, Illinois, USA) for analyzing the data. The accuracy of the final data was assessed by a qualified statistician. Demographic and socioeconomic characteristics of the participants, and participation rate of different districts of the survey area were presented using descriptive statistics. Main variables were presented as mean (SD), range or frequency and percentages. A P value of less than 0.05 was regarded as statistically significant.

\section{Discussion}

The presented protocol describes the design, methods and respiratory evaluation of the subjects in the cross sectional, population-based survey in Shiraz, Iran. We intended to provide a valid data source about CORDs via the proper quality assurance processes. The collected data from this study are supposed to indicate the prevalence of CORDs and their related determinants in the studied population. Such data could be an important advocacy tool for policy makers. They can develop targeted approaches for prevention of CORDs, and as a result have a great cost saving in their healthcare system. The biggest 
challenge of this study was the participants' drop-out. To increase the participating rate and solve the challenges at the clinic, certain approaches were implemented: the clinic was open during early morning (7:30 - 9:00 AM) and noon (12:00 - 14:00 PM) five days per week, a certain visit time of the clinic was devoted to the study participants, and spirometry costs were waived. We anticipate that future reports from the Shiraz adult respiratory disease study would estimate the prevalence of CORDs and its related determinants in the community. This study would also provide basic information about patients who should be targeted by respiratory disease prevention programs.

\section{Acknowledgments}

The authors would like to thank the deputy chancellor of Shiraz University of Medical Sciences for financial support.

\section{Footnotes}

Authors' Contribution: Seyed Masoom Masoompour contributed to the design of the study, critical revision of the content and final approval of the manuscript. Hamideh Mahdaviazad contributed to the conception and design of the study, the analysis and interpretation of data, drafting and final approval of the manuscript. Mehrab Sayadi contributed to data acquisition, drafting and final approval of the manuscript. Seiyed Mohammad Ali Ghayumi contributed to the conception and design of the study, critical revision of the content and final approval of the manuscript.

Conflict of Interest: The authors declare that they have no conflict of interest.

\section{References}

1. Ait-Khaled N, Enarson D, Bousquet J. Chronic respiratory diseases in developing countries: the burden and strategies for prevention and management. Bull World Health Organ. 2001;79(10):971-9. [PubMed: 11693980].
2. Chuchalin AG, Khaltaev N, Antonov NS, Galkin DV, Manakov LG, Antonini $\mathrm{P}$, et al. Chronic respiratory diseases and risk factors in 12 regions of the Russian Federation. Int J Chron Obstruct Pulmon Dis. 2014;9:963-74. doi: 10.2147/COPD.S67283. [PubMed: 25246783].

3. Halldin CN, Doney BC, Hnizdo E. Changes in prevalence of chronic obstructive pulmonary disease and asthma in the US population and associated risk factors. Chron Respir Dis. 2015;12(1):47-60. doi: 10.1177/1479972314562409. [PubMed: 25540134].

4. Varmaghani M, Farzadfar F, Sharifi F, Rashidian A, Moin M, MoradiLakeh M, et al. Prevalence of Asthma, COPD, and Chronic Bronchitis in Iran: A Systematic Review and Meta-analysis. Iran J Allergy Asthma Immunol. 2016;15(2):93-104. [PubMed: 27090362].

5. Varmaghani M, Rashidian A, Kebriaeezadeh A, Moradi-Lakeh M, Moin M, Ghasemian A, et al. National and sub-national prevalence, trend, and burden of asthma in Iran from 1990 to 2013; the study protocol. Arch Iran Med. 2014;17(12):804-9. [PubMed: 25481318].

6. Tazesh B, Shaabani A, Fazlollahi MR, Entezari A, Dashti R, Pourpak $Z$, Moin M. Prevalence of asthma symptoms and smoking behavior among 20 - 44 years old adults in Tehran: A telephone survey. Health. 2013;5(3):469-74. doi: 10.4236/health.2013.53064.

7. Sharifi H, Masjedi MR, Emami H, Ghanei M, Eslaminejad A, Radmand $\mathrm{G}$, et al. Burden of obstructive lung disease study in Tehran: Prevalence and risk factors of chronic obstructive pulmonary disease. Lung India. 2015;32(6):572-7. doi: 10.4103/0970-2113.168129. [PubMed: 26664162].

8. Sharifi H, Masjedi MR, Emami H, Ghanei M, Buist S. Burden of obstructive lung disease study in tehran: research design and lung spirometry protocol. Int J Prev Med. 2014;5(11):1439-45. [PubMed: 25538840].

9. Sharifi H, Masjedi MR, Emami H, Ghanei M, Eslaminejad A, Radmand $G$, et al. Interim Report from Burden of Obstructive Lung Disease (BOLD Study) in Tehran: Prevalence and Risk Factors of Chronic Obstructive Pulmonary Disease. Tanaffos. 2014;13(3):6-13. [PubMed: 25713586].

10. Statistical Center of Iran . Iran statistical yearbook. Tehran: Statistical Center of Iran Press; 2011.

11. Sichletidis L, Spyratos D, Papaioannou M, Chloros D, Tsiotsios A, Tsagaraki $\mathrm{V}$, et al. A combination of the IPAG questionnaire and PiKo-6(R) flow meter is a valuable screening tool for COPD in the primary care setting. Prim Care Respir J. 2011;20(2):184-9. doi: 10.4104/pcrj.2011.00038. [PubMed: 21597666]1 p following 189.

12. European Community Respiratory Health Survey . Variations in the prevalence of respiratory symptoms, self-reported asthma attacks, and use of asthma medication in the European Community Respiratory Health Survey (ECRHS). Eur Respir J. 1996;9(4):687-95. [PubMed: 8726932].

13. Vestbo J, Hurd SS, Agusti AG, Jones PW, Vogelmeier C, Anzueto A, et al. Global strategy for the diagnosis, management, and prevention of chronic obstructive pulmonary disease: GOLD executive summary. Am J Respir Crit Care Med. 2013;187(4):347-65. doi:10.1164/rccm.2012040596PP. [PubMed: 22878278].

14. Miller MR, Hankinson J, Brusasco V, Burgos F, Casaburi R, Coates A, et al. Standardisation of spirometry. Eur Respir J. 2005;26(2):319-38. doi: 10.1183/09031936.05.00034805. [PubMed: 16055882] 